\title{
Electrochemical and optical study of the confined aqueous layer adsorbed on gold electrodes cycled in phosphate and dodecylsulphate solutions
}

\author{
J. O. Zerbino $\cdot$ A. Maltz $\cdot$ C. Falivene $\cdot$ \\ N. E. Avaca - M. G. Sustersic $\cdot$ S. Siboni $\cdot$ \\ C. Della Volpe
}

Received: 23 June 2014/ Accepted: 14 August 2014/Published online: 27 August 2014

(C) Springer Science+Business Media Dordrecht 2014

\begin{abstract}
The properties of the confined aqueous layer potentiodynamically formed on polycrystalline gold in aqueous phosphate and sodium dodecylsulphate solutions were studied using voltammetry, ellipsometry, and contact angle measurements. The nature of the incipient oxide layer was analyzed as a function of the cycling time in the double layer-oxide monolayer potential region. The replacement of the electrolyte by hexane allows the increase of the optical signal. Different potential cycling conditions change the homogeneity of the confined aqueous incipient oxide layer leading to different structural characteristics.
\end{abstract}

Keywords Gold oxide - Ellipsometry · Contact angle · Voltammetry · Captive bubble

\section{Introduction}

The interface gold/electrolite plays a critical role in many processes, such as catalysis, membrane material growth

J. O. Zerbino $(\bowtie)$

Instituto de Fisicoquímica, INIFTA, C.C. 16, Suc. 4,

1900 La Plata, Argentina

e-mail: jzerbino@inifta.unlp.edu.ar

A. Maltz

Department Matematica, UNLP, calle 50 y 115, 1900 La Plata, Argentina

C. Falivene $\cdot$ N. E. Avaca $\cdot$ M. G. Sustersic

FICES, 25 Mayo No 384, 5730 Villa Mercedes, San Luis, Argentina

S. Siboni · C. D. Volpe

Department of Civil, Environmental and Mechanical

Engineering (DICAM), University of Trento, 38123 Trento, Italy and electrochemical sensors. It is a prototype of adsorbate/ substrate interaction. On the other hand, drops of organic solvents immiscible in water, work as a template for the production of gold nanoparticles [1-5]. Besides, the contact angle (CA) of captive drops (CD) allows the surface energy to be evaluated, as in the case of nanofiltration and the development of less fouling membrane materials $[6,7]$. The CD technique allows placing bubbles or droplets on a solid surface, where they are held captive in a cell filled with some immiscible fluid. The effect of the electrochemical potential on the surface properties of electrodes and membranes can be measured "in situ". The complete hydration of the surface avoids hydrophobic contributions to the surface tension produced by adsorbed air or empty vacuoles [8-10].

In this article, cyclic voltammetry and other potential perturbation techniques complemented with $\mathrm{CA}$ and ellipsometric measurements were employed on massive gold to characterize the interfacial region. Comparative experiments are shown, in which the metal is in contact with immiscible solvents like hexane and anisole.

This work investigates the effect of the potential cycling on the confined aqueous layer formed on gold in phosphate solutions, $\mathrm{pH}$ 6.7, and in dodecylsulphate solutions when the metal is covered by several organic immiscible solvents.

\section{Experimental}

Experiments were made at room temperature in two aqueous buffer solutions: solution $a$, a phosphate buffer solution pH $6.7\left(\mathrm{~K}_{2} \mathrm{HP}_{4} \mathrm{O}_{7} 0.25 \mathrm{M}, \mathrm{KH}_{2} \mathrm{P}_{4} \mathrm{O}_{7} 0.25 \mathrm{M}\right)$ and solution $b$, a $0.1 \mathrm{M}$ sodium dodecylsulphate solution.

Experimental conditions were similar to those described in a previous work [11-13]. The electrode was made by 
fitting axially a polycrystalline gold plaque $(99.99 \%$ purity, $1.5 \mathrm{~mm}$ thick) into a Teflon sheath. The gold plaque was then polished to a mirror finish with alumina of 0.3 and $0.05 \mu \mathrm{m}$. The sampled ellipsometric area of the electrode, vertically placed in the cell, was approximately $1 \mathrm{~mm}^{2}$ and the electrode geometric area $0.4 \mathrm{~cm}^{2}$.

The counter electrode was a gold wire placed around the working electrode. A Pt/ $\mathrm{H}_{2}$ electrode coupled to a LugginHaber capillary tip was used as a reference electrode for all potential measurements.

Ellipsometry was used illuminating the electrode with monochromatic light in the visible wavelength range $(400 \mathrm{~nm}<\lambda<700 \mathrm{~nm})$ with an incident angle of $70.128^{\circ}$. Optical data were obtained by interposing filters corresponding to five $\lambda$ (405, 450, 492, 546 and $580 \mathrm{~nm}$ ).

All experiments were performed using hydrogen reference electrode in the same electrolyte and with nitrogen bubbling. The fresh polished electrode dipped in the cell was scanned by five cycles from $E_{c}=0.04 \mathrm{~V}$ to $E_{a}=1.55$ or $1.7 \mathrm{~V}$ at a sweep rate $v=0.1 \mathrm{~V} \mathrm{~s}^{-1}$.

The experimental procedures were as follows:

\subsection{Contact angle measurements}

In the case of CA measurements a drop of solvent was introduced by a micro syringe in the cell and deposited on the gold surface polarized at open circuit voltage $E_{o c}$ and the image of the bubble was registered using optical magnification. Then, the CA was calculated with the ImageJ program and an appropriate subroutine plug-in [14-17].

\subsection{Ellipsometric measurements}

The following procedure was applied in both $a$ or $b$ solutions.

The ellipsometric parameters $\Delta$ and $\psi$, were recorded at $E_{o c}$.

\subsubsection{Procedure I}

The potential was scanned from the cathodic limit $E_{c}$ up to anodic limit $E_{a}$ at a sweep rate $v=100 \mathrm{mV} \mathrm{s}^{-1}$ during a time $\mathrm{t}$ and a new set of ellipsometric parameters $\Delta$ and $\psi$ were recorded at $E_{o c}$. The electrolyte was then evacuated and the cell was washed with distilled water and filled with hexane. Special care was taken to avoid modifying the alignment of the cell during each experiment. A new set of ellipsometric parameters $\Delta$ and $\psi$, was recorded at $E_{o c}$.

\subsubsection{Procedure II}

The same as Procedure $I$ but after the ellipsometric measurement at $E_{o c}$ the electrolyte was changed and a new set of $\Delta$ and $\psi$ data was obtained.

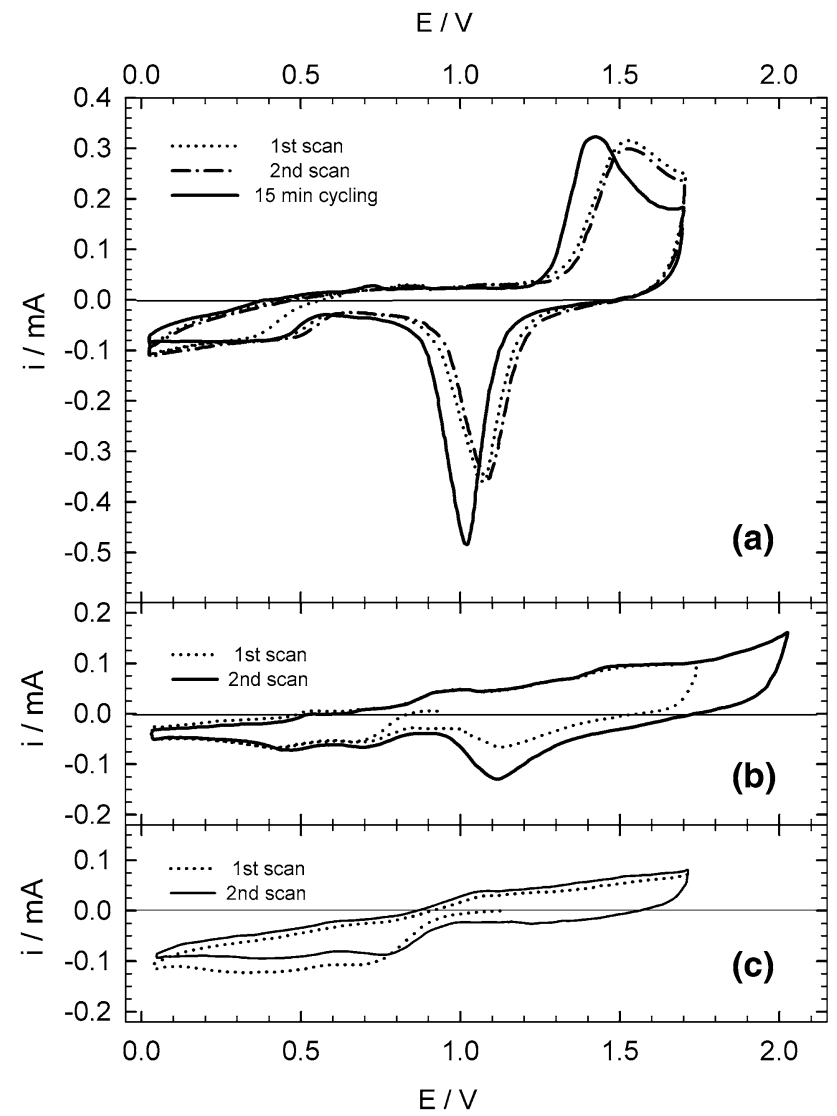

Fig. 1 Current $i$ versus potential $E$ voltammograms obtained scanning at $100 \mathrm{mV} / \mathrm{s}$ between $E c=0.04 \mathrm{~V}$ and different $E a=1.7 \mathrm{~V}$, $1.55 \mathrm{~V}$. Full line show the repetitive voltammogram observed after cycling and dotted lines show the first scans starting at Eoc. a phosphate buffer solution, b SDS solution, c SDS solution after previous 20 min cycling in buffer solution between $0.04 \mathrm{~V}$ and $1.7 \mathrm{~V}$

\section{Calculations}

The simplest model assumes a single homogeneous film. The real part of the refraction index of the film, $n$, the imaginary part of the refraction index or absorption coefficient, $k$, and the thickness, $d$, are calculated using the gradient technique [18, 19].

In the case of inhomogeneous layers, the high number of parameters makes the determination of the structure very cumbersome. However, the single homogeneous film model is a good first approximation in the description of the interface and these indices correspond to effective optical indices for the composite hydrated oxide/occluded electrolyte layer [18, 19].

The optical constants of the substrate were calculated assuming a simple water/gold interface free of optical surface layers. The $\Delta$ and $\psi$ data corresponding to the gold surface dipped in hexane can be calculated with the $n$ and $k$ values obtained in the aqueous media assuming a hexane/ gold interface free of aqueous adsorbed surface layers. 
The fitting procedure minimises the function $G$

$$
\begin{aligned}
G= & \sum\left(\Delta_{\mathrm{ij}}^{\mathrm{ex}}-\psi_{\mathrm{ij}}^{\mathrm{the}}\right)^{2}+\left(\Delta_{\mathrm{ij}}^{\mathrm{ex}}-\psi_{\mathrm{ij}}^{\mathrm{the}}\right)^{2} \text { the }- \text { size }- \text { of }- \text { the } \\
& - \text { marks }- \text { should }- \text { be }- \text { the }- \text { same }
\end{aligned}
$$

where the sub index $j$ corresponds to the optical data measured at different $\lambda_{j}$ and the sub index $\mathrm{i}$ to different deposits or thickness $d_{\mathrm{i}}$ of the confined water layer.

In the simplest process, the layer grows in thickness with constant compactness and composition bearing stable $n$ and $k$ values. The increase in the amount of optical data for a given thickness layer, e.g. measuring the $\Delta$ and $\psi$ data at different $\lambda$, leads to univocal values of thicknesses and optical constants.

The optimum theoretical $\Delta_{\mathrm{ij}}^{\text {the }}, \psi_{\mathrm{ij}}^{\text {the }}$ values are obtained minimizing the function $\mathrm{G}$ by means of a standard gradient method [20].

\section{Results}

\subsection{Voltammetric data}

Figure 1 shows the simultaneous current, $i$, and the potential, $E$, voltammograms during repetitive cycling at $0.1 \mathrm{~V} / \mathrm{s}$ with the formation (anodic scan peak) and reduction (cathodic scan peak) of the $\mathrm{Au}_{2} \mathrm{O}_{3}$ monolayer. The repetitive scans show a slight shift of the waves to more cathodic potentials, which frequently is ascribed to the cleanness of the surface and the elimination of contaminants, Fig. 1a. On the other hand, previously reported data show that during the potential cycling the oxide is not completely reduced to metallic gold and an adsorbed layer of highly hydrated incipient oxide gradually grows [12].

In solution $b$, the anodic and cathodic peaks corresponding to the oxide layer show a decrease in current related to that observed in solution $a$, which expands to higher anodic potentials indicating a strong anion adsorption, Fig. 1b. When the cycled electrode of Fig. 1a is dipped in $0.1 \mathrm{M}$ SDS solution, the voltammogram of Fig. 1c can be seen. In this case, the redox charge corresponding to the formation and reduction of the oxide layer decreases related to that observed on the fresh polished electrode.

\subsection{Contact angle measurements}

Figure 2 shows a plot of the contact angle $\theta$ versus radius $R$ of hexane and anisole drops dipped in buffer solution. The plotted radius corresponds to the immiscible drops spontaneously detached from the micro syringe dipped in the phosphate solution. For both solvents the CA shows very high values (between $162^{\circ}$ and $180^{\circ}$ ). In spite of the dispersion of the results, a tendency to reduce the angle

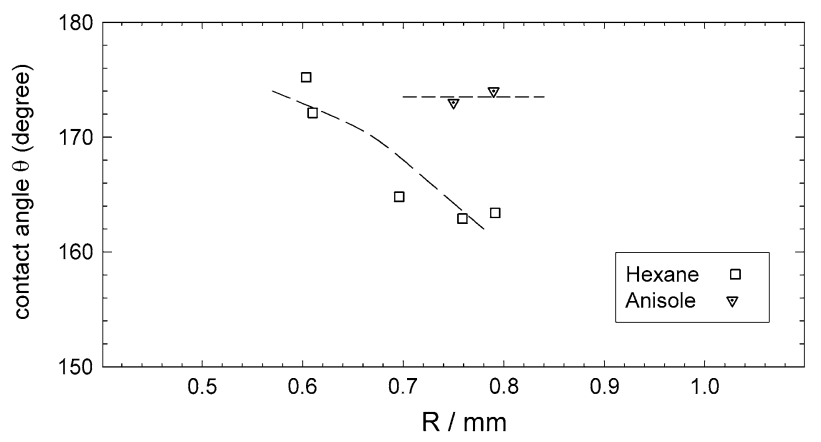

Fig. 2 Contact angle $\theta$ versus radius $R$ for drops of hexane and anisole dipped in phosphate solutions and in contact with the polished and cycled gold electrode



Fig. 3 Contact angle $\theta$ versus potential $E$ for drops of a hexane dipped in SDS solution, $R=0.4 \mathrm{~mm}, \mathbf{b}$ anisole in SDS solution, $R=0.6 \mathrm{~mm}$, and $\mathbf{c}$ anisole in phosphate solution, $R=0.7 \mathrm{~mm}$. The drops initialy are added on the polished and cycled gold electrode at the potential $E_{o c}$

with increasing radius can be observed in the case of hexane drops. The buoyancy force produces a significant deformation at the foot of the hexane drop. This effect is not observed with anisole due to the very similar density of anisole and water [21].

Figure 3 shows the effect of the electrode potential on the CA. There is no significant effect of the potential on the 


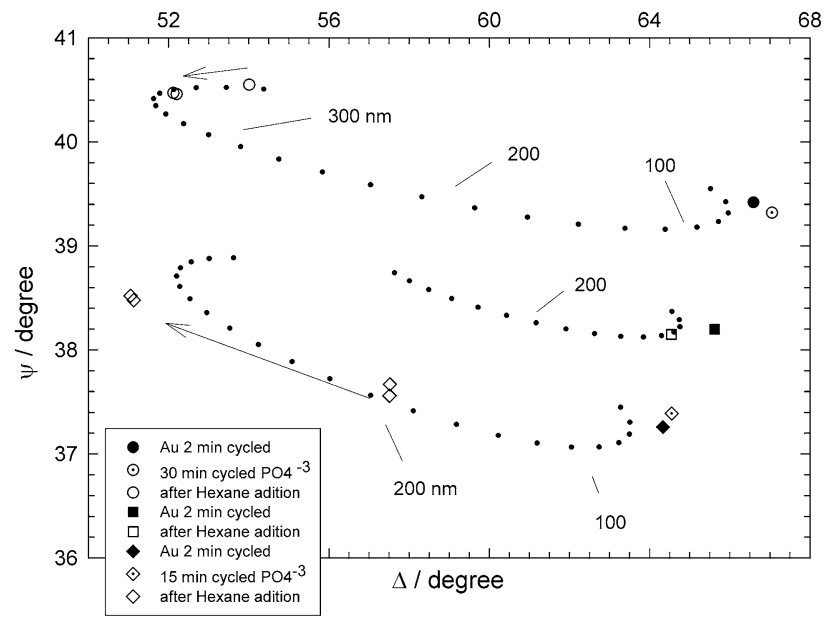

Fig. 4 Ellipsometric measurements in aqueous buffer solution obtained at $E_{o c}$ (full points), and after cycling between $0.04 \mathrm{~V}$ and $1.70 \mathrm{~V}$ for: $2 \mathrm{~min}, 15 \mathrm{~min}$ and $30 \mathrm{~min}$ in the same solution (open dotted points), and finally after replacing the electrolyte by hexane (open points)



Fig. 5 Ellipsometric measurements in aqueous SDS solution obtained at $E_{o c}$ (full points), after cycling between $0.04 \mathrm{~V}$ and $1.55 \mathrm{~V}$ for: $2 \mathrm{~min}, 20 \mathrm{~min}$ and $30 \mathrm{~min}$ in the same solution (open dotted points), and finally after the remplacing of the electrolyte by hexane (open points)

CA in the case of hexane drops immersed in both solutions $a$ (not shown) or $b$, Fig. 3a. Nevertheless, in solution $b$ a lower value of the CA $\left(146^{\circ}\right)$ related to the CA in solution $a\left(163^{\circ}\right)$ is observed due to adsorption of the anions on the hexane/water and on the gold/water interface. Moreover, in the case of anisole drops a significant decrease of the CA occurs with slow response time at potentials corresponding to the oxide layer formation, Fig. $3 b$ and $c$. The sequence of data of Fig. 3 is obtained after successive intervals of 2 min starting at the $E_{o c}$.

\subsection{Ellipsometric data}

Figures 4 and 5 show the measured $\Delta / \psi$ values corresponding to the polished electrode (full points), the values obtained after cycling (open dotted points) and those in the presence of hexane (open points). It can be noted that the change in the $\Delta$ values increases with the cycling time.

The slight differences in the $\Delta / \psi$ values corresponding to the bare electrode in the different experiments (full points in Figs. 4 and 5) are related to a window effect, that is, slight variations in the alignment of the optical cell during the electrode replacement. This systematic error modifies the calculated value of the substrate optical constants, but the resulting fitted values of both the optical constants and the thickness of the film are not altered. The dotted curves show the theoretical curves calculated for increasing thickness intervals of $20 \mathrm{~nm}$ and constant optical indices corresponding to an aqueous layer growth on the free surface of gold dipped in hexane. The arrows in Fig. 4 indicate the shift in the $\Delta / \psi$ values (open poins) measured 2 min and 12 min after having filled the cell with hexane.

\section{Discussion}

The CA experiment results shown in Fig. 2 lead to the conclusion that the gold surface retains a strongly adsorbed layer of confined water. For hexane and other solvents like butyl acetate and benzene [21], practically no changes in CA were found as a function of the applied potential in solution $a$ for holding time up to $10 \mathrm{~min}$. Even after polarization at $1.7 \mathrm{~V}$ no significant change in the CA was observed for drops of different immiscible solvents. Only in the case of anisole, and after holding times at $1.7 \mathrm{~V}$ longer than $10 \mathrm{~min}$, a systematic decrease in CA is detected. Recent work shows that the polarization at $1.7 \mathrm{~V}$ induces the formation of the gold oxide layer even for the electrode completely covered with a large drop of the solvent [21]. This is direct evidence that a confined aqueous layer remains between the metal and the immiscible solvent drop.

In the case of the air/gold/liquid interface, it is reported that different $C A$ versus $E$ data can be obtained in one experiment only starting at $E_{o c}$ and measuring the meniscus rise at potentials gradually higher than $E_{o c}$ in both directions, either cathodic or anodic to $E_{o c}$ [22]. A period of about 2 min was required to achieve a constant reading.

In the case of the measurement shown in Fig. 3 the CA decrease depends also on the previous holding time at the $0.04 \mathrm{~V} \leq E \leq 1.2 \mathrm{~V}$ potential range. The hysteresis is higher than that observed for the air/gold/liquid interface [22]. Several processes rule this behavior including the 


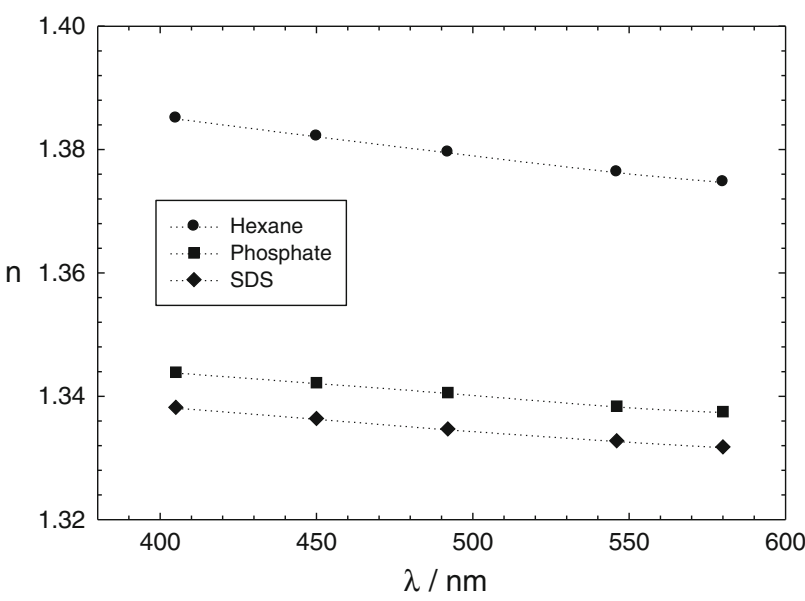

Fig. 6 Refractive index for different wavelengths of hexane, buffer phosphate and SDS aqueous solution

confined aqueous layer, anion adsorption at both the metal/ liquid and the liquid/liquid interface and the hydrogen bonds at the aqueous/anisole interface.

The Laplace equation can be applied to obtain the ideal shape of the drop in practical systems when the gravity has some perceivable effects. The Eötvös number is defined as $E o=R^{2} \Delta \rho g / 2 \gamma$, with $\gamma=$ interfacial tension, $\Delta \rho=$ density difference between the two phases, $g=$ acceleration due to gravity, and $R=$ radius of curvature of the drop at its apex. The difference of the CA measured with the spherical fitting and at the foot drop, increases for increasing Eo values and for increasing CAs [23].

For angles higher than $150^{\circ}$ the deformation becomes 3 or 4 orders of magnitude higher than for angles lower than $50^{\circ}$, assuming fixed $R, \Delta \rho$ and $\gamma$ values [23].

For the various immiscible solvents the CA lies near to $180^{\circ}$ at the foot of the drop. More work is being done to improve the accuracy in the measurements and to calculate the CA and the contact area for each solvent drop independently of buoyancy effects, in order to estimate the surface energy of the water/confined water interface [17]. The hydrogen bonded network in the thin confined aqueous layer can be distorted due to strong and long range attractive water-surface interactions [24].

Figures 6, 7 and 8 show the calculated optical indices and thicknesses of the aqueous confined layers in the wavelength optical region. The refractive indices of solutions $a$ and $b$ have similar values, while hexane shows higher optical indices, Fig. 6 [25, 26]. Previous reported data show that the incipient oxide layer grown in acid has higher refractive index than water and it increases with the cycling time. The relatively low difference in the optical indices of the confined aqueous layer and the aqueous

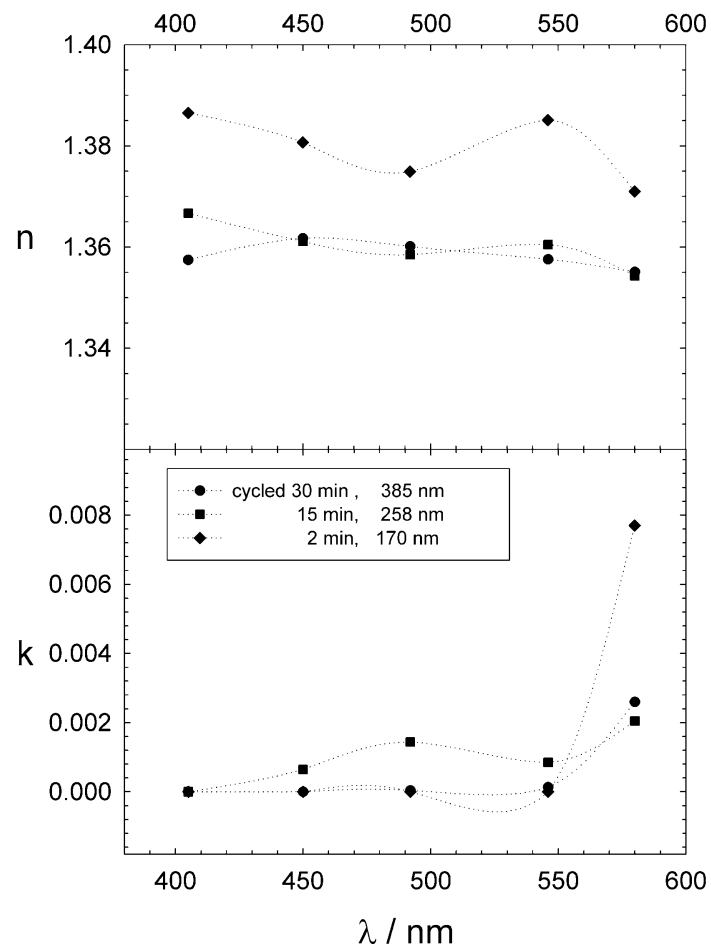

Fig. 7 Refractive and absorption indices of the confined incipient oxide layer adsorbed on gold in phosphate buffer solution after cycling between $0.04 \mathrm{~V}$ and 1.70 for $2 \mathrm{~min}$ (filled diamond), $15 \mathrm{~min}$ (filled square) and 30 min (filled circle)

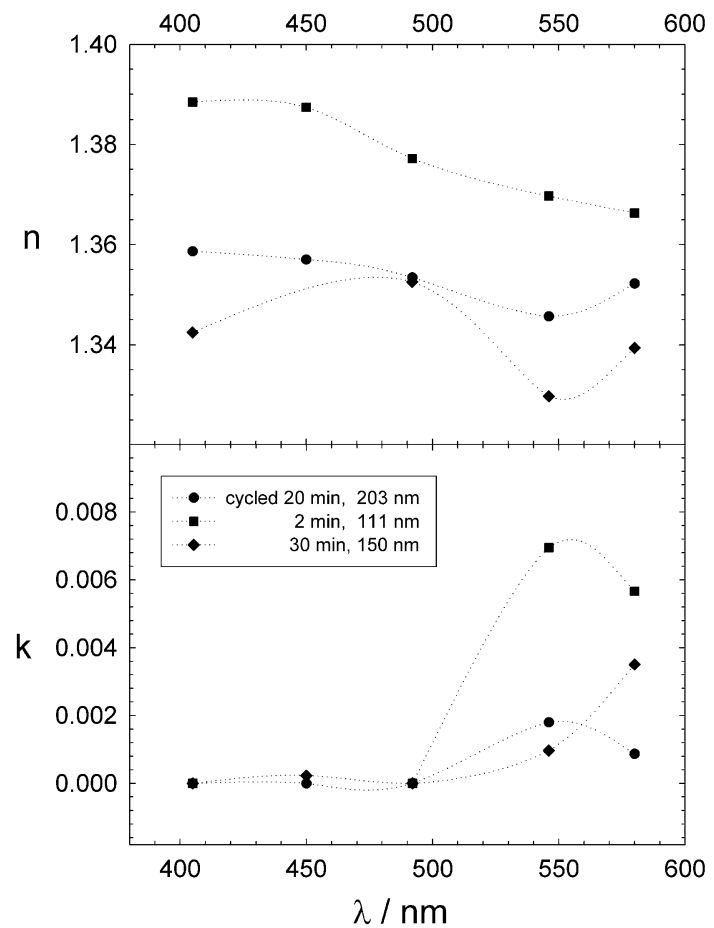

Fig. 8 Refractive and absorption indices of the confined incipient oxide layer adsorbed on gold in SDS solution after cycling between $0.04 \mathrm{~V}$ and 1.55 for $2 \mathrm{~min}$ (filled square), $20 \mathrm{~min}$ (filled circle) and $30 \mathrm{~min}$ (filled diamond) 
media produces a slight change in $\Delta / \psi$ for increasing thickness. The change of the electrolyte by hexane largely increases the ellipsometric response. In both solutions $a$ and $b$ the high fitted values of $n$ corresponding to the aqueous layer formed for a cycled time of 2 min indicate strong hexane/incipient oxide interactions.

Several investigations have reported the calculated thicknesses of the liquid/liquid interfaces. The interfacial thickness increases when the surface tension decreases, e.g. anisole/air $7.8 \AA$, water/air $4.49 \AA$ [27]. Moreover, in the case of immiscible liquid/liquid interfaces higher thicknesses are reported, e.g. hexane/water $23.50 \AA$ [28].

The "dipolar width" describes the distance required for a dielectric environment to change from one phase to another and research is devoted on modeling this region [29].

The very low compact incipient oxide/electrolyte interface can interact with the hexane/water interface. The higher hexane/water surface tension may produce a change of the initial interfacial roughness with a dynamics that involves times of more than $10 \mathrm{~min}$ to obtain stationary $\Delta /$ $\psi$ values. In the case of composite materials the effective medium theory using either Maxwell-Garnett or Bruggeman formalisms relates the optical constant of the composite fibre phase and that of the electrolyte to the volume fraction of the components providing the effective optical constants for the composite incipient oxide electrolyte.

For the low cycled gold electrode the immiscible liquid/ liquid interfaces probably attain higher roughness thicknesses comparable with an optical composite layer (aqueous incipient oxide/hexane) with effective optical index values close to those of the hexane.

\section{Conclusions}

The gold electrode is progressively covered with a highly hydrated layer of incipient gold oxide that grows in thickness and compactness with the cycling time. This adsorbed aqueous thin layer is strongly retained in contact with immiscible solvents. The replacement of the electrolyte by hexane increases the ellipsometric response. A transient of several minutes to attain the stationary optical response indicates dynamic processes at the liquid/liquid interface formed in contact with hexane. The incipient oxide layer formed after 2 min cycling probably outlines a thicker or rougher hexane/aqueous interface. Long cycling time generates a more compact hydrated incipient oxide and a flatter solvent/incipient oxide interface.
Acknowledgments This research project was supported by the "Comisión de Investigaciones Científicas de la Provincia de Buenos Aires, CIC", the "Consejo Nacional de Investigaciones Científicas y Técnicas, CONICET", the "Universidad Nacional de La Plata" and the "Universidad Nacional de San Luis".

\section{References}

1. Krishnan A, Liu YH, Cha P, Woodward R, Allara D, Vogler EA (2005) Colloids Surf B 43:95-98

2. Schramm LL, Fisher DB, Schürch S, Cameron A (1995) Colloids Surf A 94:145-159

3. Schürch S, Lee M, Gehr P (1992) Pure Appl Chem 64:1745-1750

4. Habib MA (1988) Langmuir 4:1302-1304

5. Halldorsson, Jenny (2007). Investigation of the factors influencing the wettability of conducting polymers for fluid control in microfluidic devices, $\mathrm{PhD}$ thesis, Department of Chemistry, University of Wollongong. http://ro.uow.edu.au/theses/31/

6. Azioune A, Chehimi MM, Miksa B, Basinska T, Slomkowski S (2002) Langmuir 18:1150-1156

7. Roudman AR, Digiano FA (2000) J Membr Sci 175:61-73

8. Wüstneck R, Wüstneck N, Vollhardt D, Miller R, Piso U (1999) Mater Sci Eng C 8-9:57-64

9. Teh KS, Takahashi Y, Yao Z, Lu Y-W (2009) Sens Actuators A 155:113-119

10. Myong-Hoon L (1998) Mol Cryst Liq Cryst 316:329-332

11. Zerbino JO, Pesetti LJH, Sustersic MG (2007) J Mol Liq 131-132:185-189

12. Zerbino JO, Sustersic MG (2000) Langmuir 16:7477-7481

13. Zerbino JO, Gassa L, Solid J (2003) State Electrochem 7:177-182

14. Rasband WS. Online Manual for the WCIF-ImageJ collection (1997-2004). Appendix II: Citing ImageJ and Plugins. US National Institutes of Health, Bethesda, MD. http://rsb.info.nih. gov/ij/

15. Della Volpe C, Brugnara M, Maniglio D, Siboni S, Wangdu T (2006). Contact angle, wettability and adhesion. In: Mittal KL (Ed), VSP/Brill, Leiden, vol 4, pp. 79-100

16. Brugnara M, Della Volpe C, Siboni S, Zeni D (2006). J Scanning Microsc 28:267-273

17. The ImageJ program and the appropriate plugin written by Brugnara M, Della Volpe C, and Siboni S for the calculation of contact angle may be downloaded from the following address: http://imagej.nih.gov/ij/plugins/contactangle.html

18. Jakubec K, Zdeněk Š (1985) Anal Chim Acta 172:359-364

19. Nakanishi S, Nagai T, Fukami K, Sonoda K, Oka N, Ihara D, Nakato Y (2008) Langmuir 24:2564-2568

20. Dahlquist G, Björck A (1974) Numerical Methods, Prentice-Hall

21. Gassa LM, Zerbino JO, Meyra A, Sustersic MG, Siboni S, Della Volpe C (2014) J Electroanal Chem 728: 94-110

22. Morcos I (1971) J Colloid Interface Sci 37:410-421

23. Chatterjee J (2003) J Colloid Interface Sci 259:139-147

24. Striolo A (2007) In: Balbuena PB, Seminario JM (eds) Nanomaterials: Design and simulation, Elsevier B.V

25. Hale GM, Querry MR (1973) Appl Opt 12:555-563

26. Vedam K, Limsuwan P (1978) J Chem Phys 69:4762-4771

27. Douillard JM (2009) J Colloid Interface Sci 337:307-310

28. Yang AC, Li D (1996) Colloid Surf A 113:51-59

29. Steel WH, Walker RA (2003) Nature 424:296-299 